\title{
Performance Assessment Methods of MIMO Control Systems based on Output Variance Limit
}

\author{
Jian Chu \\ Keylaboratory of Information Sensing \&Intelligent Control, \\ Tianjin University of Technology and Education, Tianjin, 300222, China \\ chujian6@126.com
}

\begin{abstract}
In this paper, performance assessment methods of multivariable control system subject to piecewise constant time varying disturbance dynamics has been 'studiet. The problem appeared on most industrial controllers are linear time invariant (LTI) while the process are often time varying. Take a series of disturbance dynamics that can be modelled by piecewise linear disturbance models, namely piecewise linear time varying (LTV) disturbance dynamics. The problem is formulated as searching for a multi-input multi-output (MIMO) benchmark control. In the paper, a method, ${ }^{\bullet}$ wich is based on output variance limit, is proposed to assess the performance of MIMotegntrol system. A weighted performance index is constructed through dividing time, vaying disturbance in to three types of constant disturbance. The upper and lower limits of the outputyariance would be determined by a special principle, and then Integral of time-weightedabsolute value of the error (ITAE) would be used to optimize the parameters of controller and give the feasible controller model. The results show that the method is simple and available in practical application.
\end{abstract}

Keywords: Limit of output variance, linear time varying disturbance, MIMO, performance assessment

\section{Introduction}

As Harris [1] firstly proposed the performance assessment in 1989, performance assessment of controller has been greatly developed. Single-input single-output (SISO) system assessment has been well dealt with, minimizing the variance of a most representative disturbance, or using the improved Type-C benchmark by the minimization of the sum of the weighted variances of all but one of major disturbances are the most common methods [2-4]. In fact, maliti-input multi-output (MIMO) control system are most widely used in practice. Early research is almost on linear time invariant (LTI) control. Under linear time invariant feedback control, the closed-loop responses are typically reflected as non-stationary time series data. Regulating of time varying disturbances would require time varying or switching control laws [5]. However, most control system in use is time variant and it would be useful to find an LTI control method to optimize time varying disturbances. Many performance indexes have been proposed, such as relative variance index (RVI) [6], on pole index [7], and so on. At the same time, many methods for performance assessment have also been proposed. For example, MIMO linear time varying (LTV) disturbance method [8-9], upper limit of output variance used in closed loop [10], qualitative and quantitive assessment method in 
simple feedback loop [11], MIMO minimum variance control method [12], variance contributions due to both disturbances and controllers method [13], obtaining a LQG benchmark based on the subspace approach [14]. However, because of the coupling and associated matrix calculation, the controller model is quite complicated and it is difficult to use in industry application.

In this paper, standard and improved Type-C benchmarks used in SISO system are extended as the regular linear time varying disturbance (LTVD) benchmark and weighted LTVD benchmark, respectively. The regular LTVD benchmark aims at minimizing the total output variance while the weighted LTVD benchmark at the aim of minimization of the sum of the weighted total variances. For a new defined generalized LTVD problem, the objective is to limit the output variance to assess the performance of MIMO LTVD system.

The main contributions of this paper are development of a new LTVD benchmark which is ${ }^{\bullet}$ based on the controller parameter with output variance limit. In the paper, varying disturbance is divided in to three types of constant disturbance and a weighted performanee index is constructed. After specifying the reasonable output variance, the upper and lower bounds of the generalized multivariable system closed-loop output variance could be obtained through the calculation of diagonal interactors, and then, controller parameters with minimum variance can be achieved. The remainder of the paper is organized as follows: Multivariate controller Multivariate controller performance assessment is rexisited in Section 2. The LTVD benchmark problems based on output variance limit for MIMO processes are derived in Section 3, and they are referred to as the regular, weighted and generalized LTVD benchmark problems, respectively. The solutions to these LTVD benchmark problems are given in Section 4.

\section{Review of Multivariate Controlier Performance Assessment}

Lots of research [18-20] have proposed solutions for the performance assessment of multivariate controllers based on the minimum variance control (MVC) benchmark. Firstly in the section, the interactor matrix would be introduced, and then filtering and correlation analysis (FCOR) algorithm would be summarized.

\subsection{Interactor matrix}

Multivariate process can be represented by

$$
\text { 2. } \Phi_{t}=t\left(q^{-1}\right) u_{t}+N\left(q^{-1}\right) a_{t}
$$

where $T\left(q^{-1}\right)$ is a $p p \times m$ proper, rational polynomial transfer function matrix, $N(q-1)$ is a disturbance model $\frac{T}{h}, u_{t}$ and $a_{t}$ are output, input and white noise vectors, then there exists a unique, non-singuar, $p \times p$ lower left triangular polynomial matrix D, such that $|D|=q^{r}$ and

$$
\lim _{q^{-1} \rightarrow 0} D T=\lim _{q^{-1} \rightarrow 0} \tilde{T}=K_{0}
$$

where $K_{0}$ is a full rank (full column rank or full row rank) constant matrix, the integer $\mathrm{r}$ is defined as the number of infinite zeros of $\mathrm{T}$, and $\tilde{T}$ is the time delay free transfer function (factor) matrix of $\mathrm{T}$ which contains only finite zeros. The matrix $\mathrm{D}$ is defined as the interactor matrix and can be written as

$$
D=D_{0} q^{d}+D_{1} q^{d-1}+D_{2} q^{d-2}+\ldots+D_{d-1} q
$$


where $\mathrm{d}$ is denoted as the order of the interactor matrix and is unique for a given transfer function matrix, and $D_{i} s$ (for $\left.i=0,1, \ldots, d-1\right)$ are coefficient matrices[21]. If the interactor matrix satisfies

$$
D^{T}\left(q^{-1}\right) D(q)=1
$$

Then this interactor matrix is defined as the unitary interactor matrix which has been established by Peng and Kinnaert [22]. This unitary interactor is an all-pass term, and factorization of such a unitary interactor matrix does not change the spectral property of the system. The interactor matrix is an equivalent form of the time delay in multivariate systems and needs process model to capture the delay terms. The interactor matrix can be factored out by using QR factorization or singular value decomposition or direct definition [23].

\subsection{Conventional algorithm with MVC benchmark}

With unitary interactor matrix D, the process output in (1) can be expressed as

$$
\Phi_{t}=T\left(q^{-1}\right) u_{t}+N\left(q^{-1}\right) a_{t}=D^{-1} T u_{t}+N a_{t}
$$

Since

$$
q^{-d} D N=F+q^{\wedge} R,
$$

The interactor filtered output can be written as

$$
\Phi_{t}=q^{-d} D y_{t}=q^{-d} \tilde{T} u_{t}+q^{2} D N a_{t}=F a^{-}+q\left(\tilde{T} u_{t}+R a_{t}\right)
$$

Then the closed-loop response of the filtered output under minimum variance control can be acquired as

$$
\Phi_{t} \sim F a_{t}=\left(F_{0}+F_{1} q^{-j}+\ldots+F_{d-1} q^{-d+1}\right) a_{t}
$$

This is the feedback control invartant tern, and it can be solved as

$$
\Phi_{t \mid m v}=Q D^{-1}\left(F_{0}+F_{1} q^{-1}+\cdots+F d_{-1} q^{-d+1}\right) a_{t}
$$

Since

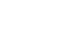

Therefore

$$
\begin{aligned}
& \Phi_{t \mid m v}=\left(D_{0}^{T}+\ldots+D_{d-1}^{T} q^{d-1}\right)\left(F_{0}+F_{1} q^{-1}+\ldots+F_{d-1} q^{-d+1}\right) a_{t} \\
& \stackrel{4}{=}\left(E_{0}+E_{l} q^{-1}+\ldots+E_{d-1} q^{-d+1}\right) a_{t}
\end{aligned}
$$

where

$$
\left(E_{0}, E_{1}, \ldots, E_{d-1}\right) \stackrel{\Delta}{\Delta}\left(D_{0}^{T}, D_{1}^{T}, \ldots, D_{d-1}^{T}\right)\left(\begin{array}{ccccc}
F_{0} & F_{1} & \ldots & F_{d-2} & F_{d-1} \\
F_{1} & F_{2} & \ldots & F_{d-1} & \\
\ldots & \ldots & & & \\
\ldots & F_{d-1} & & & \\
F_{d-1} & & &
\end{array}\right)
$$




$$
E\left(a_{t} a_{t}^{T}\right)=\sum_{a}
$$

then

$$
\left.E\left(\Phi_{t} \Phi_{t}^{T}\right)\right|_{n v}=\operatorname{trace}\left(E_{0} \sum_{a} E_{0}^{T}+E_{l} \sum_{a} E_{l}^{T}+\ldots+E_{d-l} \sum_{a} E_{d-l}^{T}\right)
$$

The overall performance indices can thus be calculated as the following:

$$
\eta_{0}=\frac{\operatorname{trace}\left(\left.E\left(\Phi_{t} \Phi_{t}^{T}\right)\right|_{n v}\right)}{\operatorname{trace}\left(E\left(\Phi_{t} \Phi_{t}^{T}\right)\right)}
$$

The individual performance indices can thus be calculated as the following:

$$
\eta_{i}=\frac{\operatorname{diag}\left(\left.E\left(\Phi_{t} \Phi_{t}^{T}\right)\right|_{m v}\right)}{\operatorname{diag}\left(E\left(\Phi_{t} \Phi_{t}^{T}\right)\right)}
$$

An important assumption of these existing results with minimun variance control as the benchmark is that the disturbance dynamics are lineap time invariant. However, practical experiences indicate that the disturbance dynamics are often time varying. In the following sections, we will consider a class of time varying disturbance dynamics and then present our solutions for their regulatory performance assessment problem.

\section{LTVD Benchmarks for MIMO Processes}

\section{1 process description}

We firstly state the following assumptions: (1) The plant is linear time invariant with no unstable zeros. (2) The disturbance model is piecewise linear time varying. (3) The controller is time invariant.

Consider a MIMO process $T\left(q^{-1}\right)$ shown in Figure 1 subject to piecewise constant disturbance dynamics. Time varying disturbance matrix $N_{t}\left(q^{-1}\right)$ varies at different time, and it can be expressed by piecewise constant disturbance. Error weighted matrix Pc and control weighted matrix Fc can limit the maximum value of control signal so as to make the controller more feasible. Matgix $N_{t}\left(q^{-1}\right)$ of multi variance control system can be acquired from a set of specific value, operation parameter of the controller or output of closed loop. Linear controller $Q\left(q^{-1}\right)$ also have influence on the couple part of the controlled system $G\left(q^{-1}\right)$. White noise sequences with-zero mean.

We have assumed that disturbance dynamics are piecewise linear time varying. This type of time varying disturbances are reflected as different output trajectories in different time sections, each section having a linear time invariant disturbance model. It is assumed that the $\mathrm{i}$-th disturbance is the most representative of the disturbances that are affecting the process while the $\mathrm{j}$-th section of the disturbances corresponds to the significant but transient upset within the process. It is required that the closed-loop response to the $\mathrm{j}$-th section of disturbance is to be settled down along some user specified trajectory. The process is also affected by some other disturbances at different time periods beside the two kinds referred before. We denote the $\mathrm{k}$ - th disturbance as the $\mathrm{k}$ - th section response or simply call it as the kth section for simplicity. The k-th disturbance has the middle significance between $\mathrm{i}$-th and $\mathrm{j}$ th, it is just used to assess whether the performance can meet the demand after the adjustment of $\mathrm{j}$-th disturbance. In both linear time variant system and linear time invariant system, the 
important kinds of the disturbance are i-th and j-th, k-th can be partly included in i-th and the other part in $\mathrm{j}$-th..

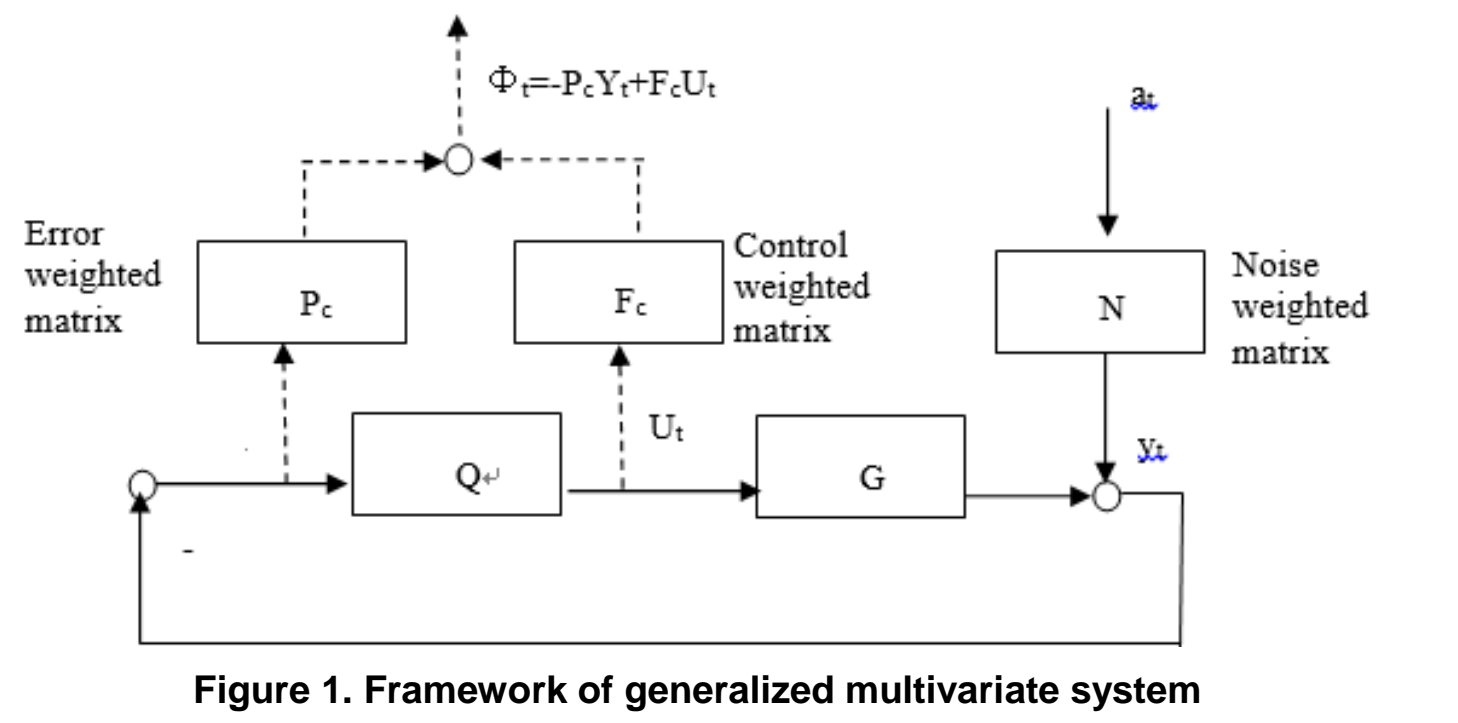

In order to make $\mathrm{j}$-th disturbance meet the demand of output variance, the transfer function $T\left(q^{-1}\right)$ can be used to instead of the feedback term. Usually, the $T\left(q^{-1}\right)$ can be expressed as the following:

Where
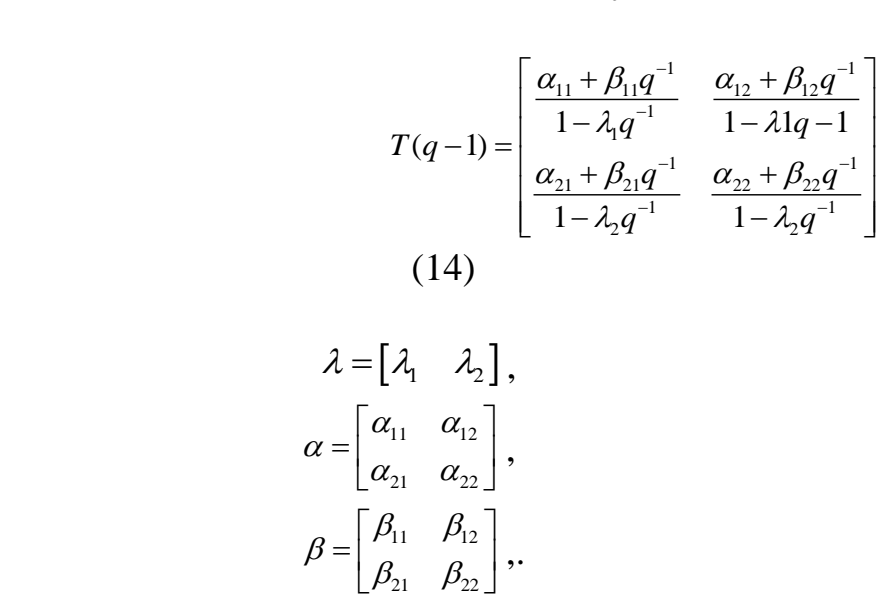

$\alpha, \beta$ are parameters to determined, and $\lambda$ represents the response dynamics that can be specified by the user 24$]$. The closed loop output of the $\mathrm{j}$-th disturbance can be expressed as the following

$$
\Phi_{t}^{(j)}=F_{j}\left(q^{-1}\right) a_{t}+q^{-d G} T\left(q^{-1}\right) a_{t}
$$

Where, $F_{j}$ is constant feedback control term separated from generalized matrix though Diophntine function..

$$
N_{G}^{(j)}=F_{j}\left(q^{-1}\right)+q^{-d G} G_{R}\left(q^{-1}\right)
$$

$d_{G}$ is the highest order of Delay matrix of I/O in the controlled system. The closed loop output of the i-th disturbance and k-th can be expressed as the following respectively:

$$
\begin{aligned}
& \Phi_{t}^{(\mathrm{i})}=\left[F_{i}\left(q^{-1}\right)+q^{-d G} T\left(q^{-1}\right)\right] N_{j}^{-1} N_{i} a_{t} \\
& \Phi_{t}^{(\mathrm{k})}=\left[F_{k}\left(q^{-1}\right)+q^{-d G} T\left(q^{-1}\right)\right] N_{j}^{-1} N_{k} a_{t}
\end{aligned}
$$


We can know output total variance of closed loop is equal to the $H_{2}$ norm of the transfer matrix from the Parseval principle, so

$$
\begin{aligned}
& \operatorname{var}\left(\Phi_{t}^{(\mathrm{j})}\right)=\left\|\left[F_{j}\left(q^{-1}\right)+q^{-d G} T\left(q^{-1}\right)\right]\right\| \\
& \operatorname{var}\left(\Phi_{t}^{(\mathrm{i})}\right)=\left\|\left[F_{j}\left(q^{-1}\right)+q^{-d G} T\left(q^{-1}\right)\right] N_{j}^{-1} N_{i}\right\| \\
& \operatorname{var}\left(\Phi_{t}^{(\mathrm{k})}\right)=\left\|\left[F_{j}\left(q^{-1}\right)+q^{-d G} T\left(q^{-1}\right)\right] N_{j}^{-1} N_{k}\right\|
\end{aligned}
$$

According to the optimization of free parameters of transfer function $T\left(q^{-1}\right)$, the best control model can be expressed as the following:

$$
Q_{G}=\frac{G_{R}\left(q^{-1}\right)-L_{R}\left(q^{-1}\right)}{q^{d G}\left[F_{c}-P_{c} G\left(q^{-1}\right)\right]\left[F_{j}\left(q^{-1}\right)+q^{-d G} T\left(q^{-1}\right)\right]}
$$

\section{2 determination of the upper and lower limit of the variance}

As described before, two free parameters are included in the transfer matrix $T\left(q^{-1}\right)$. For generalized multi-variances system, the two parameters can be calculated though integral of time-weighted absolute value of the error (ITAE) optimization:

$$
J_{\text {IATE }}=\int_{0}^{\infty} t\left|e_{G}(t)\right| d t
$$

Where $e_{G(t)}$ is the generalized error of system which is relative with $T\left(q^{-1}\right)$. At least one group value of $\alpha$ and $\beta$ would be acquired to make the trace of closed loop output variance under the i-th and k-th disturbance, and Variance of $\mathrm{j}$ (h disturbance also can be limited in a range, but it should be given some constraints to the variance of $j$-th disturbance based on the fact. Here, we can determine the lower limit value of 1 closed loop output variance: the sum of variance of the three kinds of distlurbance.

When we calculate the lower value of the multi-variance system, the significance of the disturbance on the system should be paid attention. The output variance of i-th disturbance cannot be avoid in the practice, and the value should be smaller and smaller. Because of the random chance, $\mathrm{j}$-th is the major reason of the deterioration of the performance. It should be ensured to keep the track of previous trajectory. If the output variance of $\mathrm{j}$-th is too small, idealized $\alpha$ and $\beta$ value would be got and it is quite difficult to get the proper control parameters. Since k-th disturbance is included in $\mathrm{i}$-th and $\mathrm{j}$-th disturbance, so output variance of i-th and j-th disturbance should represent different performance. This can be resolved by giving different eifght $\rho_{i}$ and $\rho_{j}$ to them where $\rho_{i}$ and $\rho_{j}$ represent different percentage of $\mathrm{k}$-th included in - th and $\mathrm{j}$-th.

In most conditions, the upper limit of output variance calculated would be less than the actual output error, so the calculated value of the upper limit of output variance can be used as the upper limit. Here, we set the upper limit of output variance of the generalized system as:

$$
\left[\min \left[\rho_{i} \cdot \operatorname{var}\left(\Phi_{t}^{(i)}\right)+\rho_{k} \cdot \operatorname{var}\left(\Phi_{t}^{(k)}\right)\right]+\operatorname{var}\left(\Phi_{t}^{(j)}\right), V\right]
$$

Where, $\mathrm{V}$ is the user defined upper limit matrix. It has two advantages to control the output variance. Firstly, if a bigger upper limit of output variance is set, it would get a higher productivity, or else, it would get a higher quality; secondly, reasonable upper and lower limit of output variance can improve the efficiency with the feasibility of the controller. 


\section{3 calculation of MIMO performance assessment}

Diagonal interactor matrix (DIM) would be used in the calculation. We just need the disturbance model and delay matrix to calculate the $\operatorname{var}_{\text {opt }}\left(\Phi_{t}\right)$ [25]. It can avoid the system error when identify the interactor matrix (IM). Although there are some differences between the two methods, it is easy to prove the limit of the relative error according to the following formula.

$$
e=\frac{\operatorname{var}_{o p t}^{D I M}\left(\Phi_{t}\right)-\operatorname{var}_{o p t}^{I M}\left(\Phi_{t}\right)}{\operatorname{var}\left(\Phi_{t}\right)}
$$

And $\operatorname{var}\left(\Phi_{t}\right)$ is quadratic term, so formula is absolute convergent

$$
\sum_{t=1}^{n} e=\sum_{t=1}^{n} \frac{\operatorname{var}_{o p t}^{D I M}\left(\Phi_{t}\right)-\operatorname{var}_{o p t}^{I M}\left(\Phi_{t}\right)}{\operatorname{var}\left(\Phi_{t}\right)}
$$

The assessment index of the generalized multi-variance system can be defined as

$$
\eta=\frac{\operatorname{trace}\left[\operatorname{var}_{\text {opt }}\left(\Phi_{t}\right)\right]}{\operatorname{trace}\left[\operatorname{var}_{a c t}\left(\Phi_{t}\right)\right]}, \quad \eta \in[0,1]
$$

If $\eta$ trended towards 1 , it means the performance is better, otherwise, the performance is worse and it needs optimize the control parameters.

\section{Simulation example}

The following MIMO system is considered to investigate the different cases of the LTVD benchmark problems.

The process transfer matrix and the odntroller are given respectively by

$$
\left.\begin{array}{cc}
\frac{q^{-1}}{1-0.4 q^{-1}} & \frac{q^{-2}}{1-0.1 q^{-1}} \\
0.3 q^{-1} & \frac{q^{-2}}{1-0.8 q^{-1}}
\end{array}\right],\left[\begin{array}{cc}
\frac{0.5-0.2 q^{-1}}{1-0.5 q^{-1}} & 0 \\
0 & \frac{0.25-0.2 q^{-1}}{1-0.25 q^{-2}}
\end{array}\right]
$$

The process is assumed to be affected by three piecewise constant disturbance dynamics with the models proyided below with respect to three different disturbances:

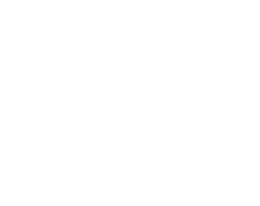

$$
\begin{aligned}
& N_{1}=\left[\begin{array}{cc}
\frac{1}{1-0.5 q^{-1}} & \frac{-0.6 q^{-1}}{1-0.5 q^{-1}} \\
\frac{0.5 q^{-1}}{1-0.5 q^{-1}} & \frac{1}{1-0.5 q^{-1}}
\end{array}\right], \quad t \in[1,2001) \\
& N_{2}=\left[\begin{array}{ll}
\frac{1}{1-0.9 q^{-1}} & \frac{-0.6 q^{-1}}{1-0.9 q^{-1}} \\
\frac{0.5 q^{-1}}{1-0.9 q^{-1}} & \frac{1}{1-0.9 q^{-1}}
\end{array}\right], \quad t \in[2001,3001)
\end{aligned}
$$




$$
N_{3}=\left[\begin{array}{cc}
\frac{1}{1-0.7 q^{-1}} & \frac{-0.6 q^{-1}}{1-0.7 q^{-1}} \\
\frac{0.5 q^{-1}}{1-0.7 q^{-1}} & \frac{1}{1-0.7 q^{-1}}
\end{array}\right], \quad t \in[3001,4500]
$$

The second disturbance has the slowest dynamics and is considered here as the major disturbance. Control of the second disturbance needs to satisfy the user specified closed-loop response. As descripted in section 3, k-th disturbance would be included in i-th and $\mathrm{j}$-th disturbance and the value of $\rho \mathrm{i}$ and $\rho \mathrm{j}$ would be set as 0.95 and 0.05 respectively. The major disturbance $N_{2}+0.05 \times N_{3}$ can be factorized from Diophantine equation as

$$
F=\left[\begin{array}{cc}
-0.9603 q^{-1} & -0.2861 q^{-1} \\
-0.2903+0.2273 q^{-1} & 0.9529+1.043 q^{-1}
\end{array}\right]
$$

For the performance assessment problems, $\operatorname{var}_{o p t}\left(\Phi_{t}{ }^{2}\right)$ represents the total variance and $\eta$ is the corresponding performance index.

\section{1 performance calculation}

If the process and disturbance models are known, the optimal output total variances can be calculated with different user chosen $\lambda$ values, based on the regular, weighted and generalized LTVD benchmarks, respectively. The results are given in Tables 1-3 respectively.

\begin{tabular}{|c|c|c|c|c|c|}
\hline$\lambda$ & $\alpha$ & $N$ & $\mathrm{Pr}_{o p t}\left(\Phi_{t}^{1}\right)$ & $\operatorname{var}_{o p t}\left(\Phi_{t}^{2}\right)$ & $\operatorname{var}_{o p t}\left(\Phi_{t}^{3}\right)$ \\
\hline$[0.3,0.3]$ & {$\left[\begin{array}{cc}-0.3007 & -0.07 \\
0.0535 & 0.47\end{array}\right.$} & $\frac{-0.0251}{0.2419}$ & 2.7938 & 3.6753 & 3.0154 \\
\hline$[0.5,0.5]$ & {$\left[\begin{array}{cc}-0.3239 & -0.0934 \\
0.0384 & 0.5120\end{array}\right]$} & {$\left[\begin{array}{cc}-0.2244 & -0.0001 \\
0.0290 & 0.1967\end{array}\right]$} & 2.7223 & 3.8844 & 3.0028 \\
\hline$[0.7,0.7]$ & {$\left[\begin{array}{cc}-0.3542 & -0.0787 \\
0.0210 & 0.5695\end{array}\right]$} & {$\left[\begin{array}{cc}0.0818 & -0.0426 \\
0.0095 & 0.1291\end{array}\right]$} & 2.6298 & 4.3277 & 2.9846 \\
\hline
\end{tabular}

\section{Table 1. Performance limit results based on the regular LTVD benchmark}

From Table 1 to Table 3, with the increasing $\lambda$ values, the optimal total variance of the first and third section is getting smaller, while the optimal total variance of the second section is becoming Targer. If the $\lambda$ values are too small, the output variance of $\mathrm{i}$-th and $\mathrm{k}$-th disturbance may be too small, and it would make the performance of system quite worse. At the same time, control performance of $\mathrm{j}$-th would be better with the increasing of $\lambda$ values, and it may get false result of bad system performance but good assessment. Compared with the references, the calculation method proposed in the paper can reflect the sensitivity of system performance with the change of $\lambda$ values and it can help to determine the range of $\lambda$ values. 
Table 2. Performance limit results based on the weighted LTVD benchmark

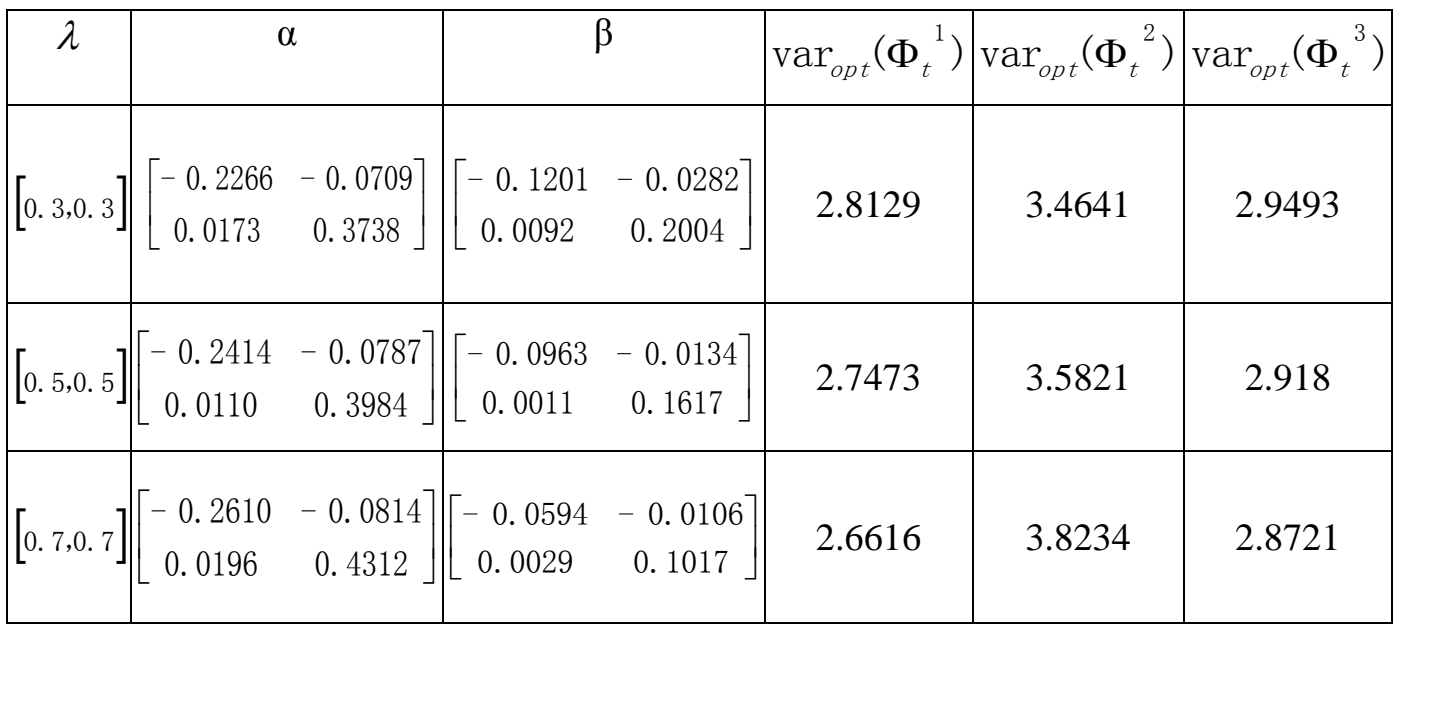

Table 3. Performance limit results based on the generalized LTVD benchmark

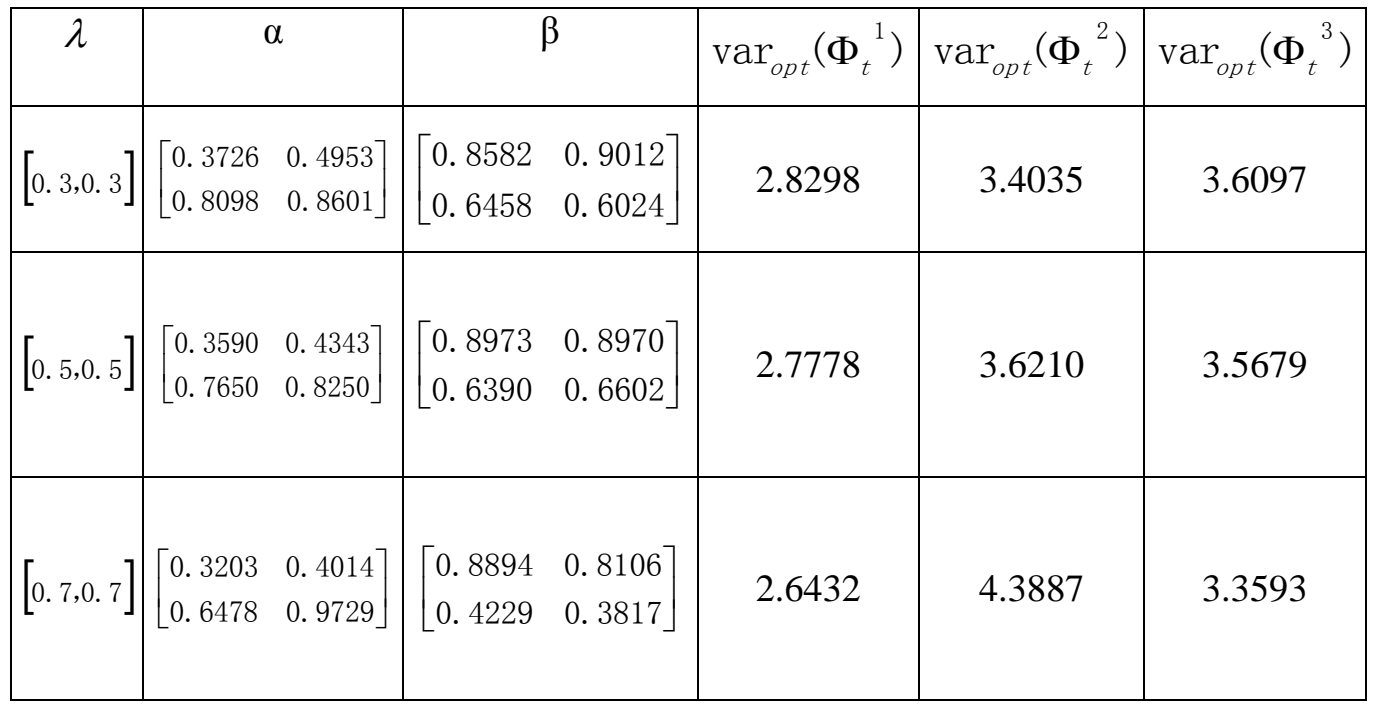

From the Table 4. we can see that when $\rho_{i}$ and $\rho_{j}$ have been set 0.95 and 0.9 respectively, the performance index is much better than before. For the reason of taking k-th into consideration, it is good for the track of optimal performance. Keeping the $\rho_{i}$ and $\rho_{j}$ as constant, when the upper value is limited at 14 , it would have a higher performance demand of system, and the performance index would be smaller, or else, the performance index would be higher. So, it must be adjust the control parameters based on the practice use. 
Table 4. Performance assessment results with output variance limit

\begin{tabular}{|c|c|c|c|c|c|c|}
\hline$\left[\rho_{i}, \rho_{j}\right]$ & $\begin{array}{l}\text { Limit } \\
\text { value }\end{array}$ & $\alpha$ & $\beta$ & $\operatorname{var}_{o p t}\left(\Phi_{t}{ }^{1}\right) / \eta$ & $\operatorname{var}_{o p t}\left(\Phi_{t}^{2}\right) / \eta$ & $\operatorname{var}_{o p t}\left(\Phi_{t}^{3}\right) / \eta$ \\
\hline$[0.9,0.1]$ & \begin{tabular}{|c|}
{$[12.7$} \\
$15.7]$
\end{tabular} & {$\left[\begin{array}{cc}-0.7442 & -0.8322 \\
0.7088 & 0.6801\end{array}\right]$} & $\mid\left[\begin{array}{cr}-0.4233 & -0.5377 \\
0.4739 & 0.6342\end{array}\right]$ & $\begin{array}{l}3.1508 \\
/ 0.9707\end{array}$ & $\begin{array}{l}4.0388 \\
/ 0.4976\end{array}$ & $\begin{array}{l}3.4996 \\
/ 0.8069\end{array}$ \\
\hline$[0.95,0.05]$ & {$\left[\begin{array}{c}11.85 \\
15.7]\end{array}\right.$} & {$\left[\begin{array}{cc}-0.7590 & -0.4943 \\
0.8670 & 0.8468\end{array}\right]$} & {$\left[\begin{array}{cc}-0.8556 & 0.8930 \\
0.6424 & 0.6002\end{array}\right]$} & $\begin{array}{l}3.2507 \\
/ 0.9938\end{array}$ & $\begin{array}{l}6.2456 \\
/ 0.7685\end{array}$ & $\begin{array}{l}3.9764 \\
/ 0.9147\end{array}$ \\
\hline$[0.95,0.05]$ & {$\left[\begin{array}{c}11.85 \\
14]\end{array}\right.$} & 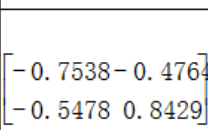 & {$\left[\begin{array}{cc}-0.3094 & 0.7530 \\
0.4529 & 0.5787\end{array}\right]$} & $\begin{array}{l}3.1571 \\
/ 0.9686\end{array}$ & $\begin{array}{l}5.1061 \\
0.6287\end{array}$ & $\begin{array}{l}3.5782 \\
/ 0.8352\end{array}$ \\
\hline
\end{tabular}

\section{2 performance assessment calculation}

Performance index under the three kinds of disturbances is shown an Figure 2-4.

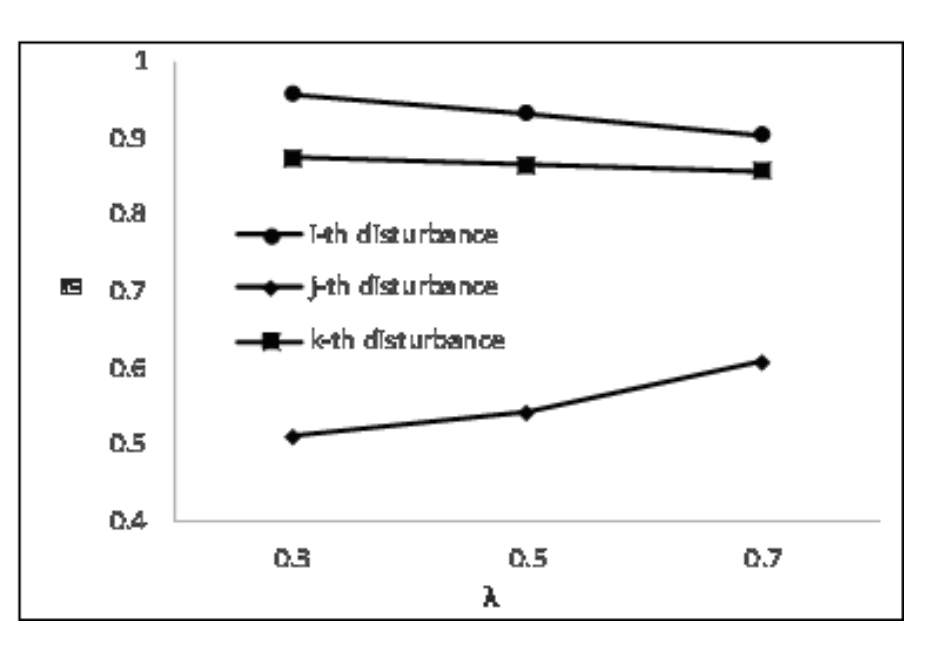

Figure 2. Performance index of the regular LTVD benchmark

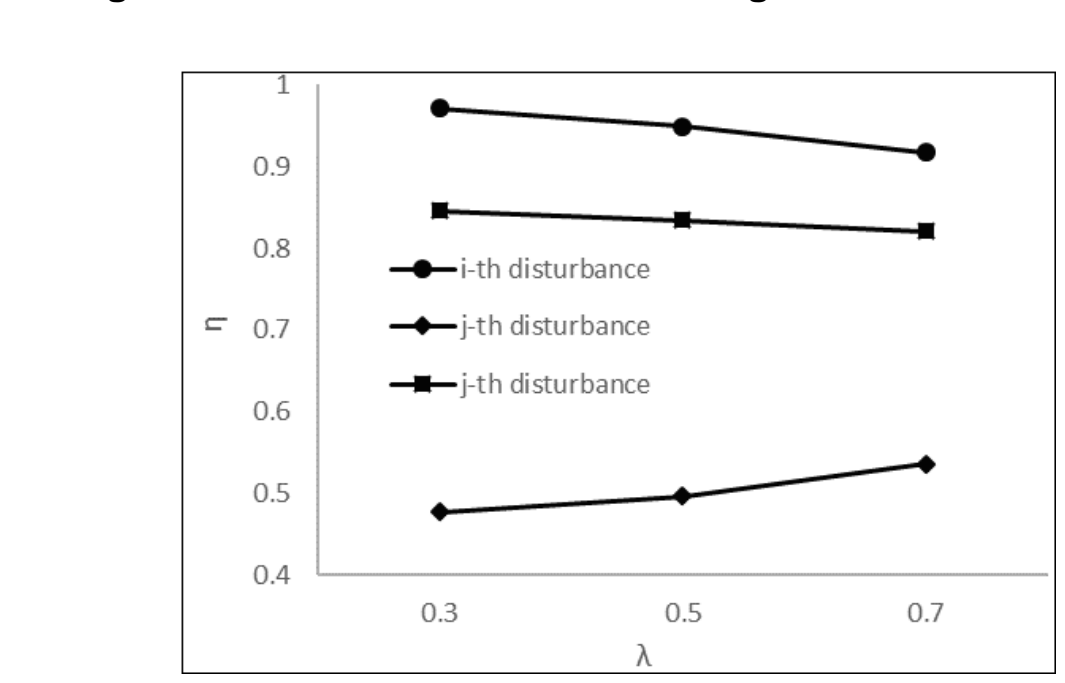

Figure 3. Performance index of the regular LTVD benchmark 


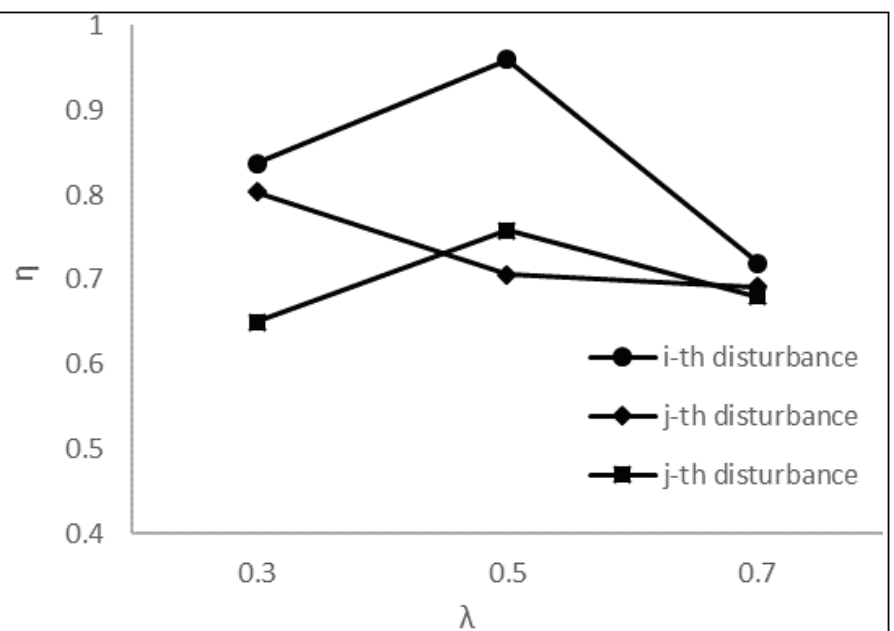

Figure 4. Performance index of the regular LTYD benchmark

\section{Conclusion}

Three LTVD benchmarks for MIMO processes are studied in this paper for evaluating the controller performance. The process is subject to piecewise constant LTV disturbance dynamics. Property of each kind of disturbance and its influence on the loop are analyzed, and upper and lower limits of weighted outputvariance are used to control output variance of closed loop; according to the ITAE optimization, we can determine the parameters of the controller. This method can give adviee to adjust the value' of parameters and meet the use's demand.

\section{References}

[1] T. J. Harris, “Assessment of control loop performance”, The Canadian Journal of Chemical Engineering, vol. 67 , no. 5, (1989), pp. 856-861.

[2] F. Olaleye, B. Huang and E. Tamayo, Incustrial applications of a feedback controller performance assessment of time-variant processes", Industrial \& Engineering Chemical Research, vol. 43, (2004), pp. 597-607.

[3] F. Olaleye, B. Huang and E. Tamayo, "Performance assessment of control loops with time-variant disturbance dynamics", Journa of Process Control, vol. 14, no. 8, (2004), pp. 867-877.

[4] F. Xu and B. Huang, "Pefornance monitoring of SISO control loops subject to LTV disturbance dynamics: An improved LTI benchmark", Journal of Process Control, vol. 16, no. 6, (2006), pp. 567-579.

[5] Z. Sun and S. S. Ge, "Analysis and synthesis of switched linear control systems", Automatica, vol. 41, (2005), pp. 181-195.

[6] S. Bezergianni and C. Georgakis, "Controller performance assessment based on minimum and open-loop output variance), Control Engineering Practice, vol. 8, (2000), pp. 791-797.

[7] A. Horch and A. J. Isaksson, "A modified index for control performance assessment", Journal of Process Control, yol. 9, no. 6, (1999), pp. 475-483.

[8] F. Xu and B. Huang, "Performance monitoring of SISO control loops subject to LTV disturbance dynamics: An improved LTI benchmark", Journal of Process Control, vol. 16, no. 6, (2006), pp. 567-579.

[9] F. Xu, B. Huang and E. C. Tamayo, "Performance assessment of MIMO control systems with time-variant disturbance dynamics", Computers and Chemical Engineering, vol. 32, (2008), pp. 2144-2154.

[10] F. Xu, K. H. Lee and B. Huang, "Monitoring control performance via structured closed-loop response subject to output variance/covariance upper bound", Journal of Process Control, vol. 16, no. 9, (2006), pp. 971-984.

[11] K. J. Astrom, "Assessment of achievable performance of simple feedback loops", International Journal of Adaptive Control and Signal Processing, vol. 5, (1991), pp. 3-19.

[12] T. Harris, F. Boudreau and J. F. MacGregor, "Performance assessment using of multivariable feedback controllers", Automatica, vol. 32, (1996), pp. 1505-1518. 
[13] L. Desborough and T. Harris, "Performance assessment measures for univariate feedforward/feedback control”, Canadian Journal of Chemical Engineering, vol. 71, (1993), pp. 605-616.

[14] C. Dai and S. H. Yang, "Controller performance assessment with a LQG benchmark obtained by using the subspace method", In Proceedings of the control 2004. UK: University of Bath, ID-066, (2004).

[15] X. Wang, B. Huang and T. Chen, "Multi rate minimum variance control design and control performance assessment: a data driven subspace approach", IEEE Transaction on Control System Technology, vol. 15, no. 1, (2007), pp. 65-74.

[16] F. Olaleye, B. Huang and E. Tamayo, "Performance assessment of control loops with time-variant disturbance dynamics", Journal of Process Control, vol. 14, no. 8, (2004), pp. 867-877.

[17] C. A. McNabb and S. J. Qin, "Projection based MIMO control performance monitoring: I-covariance monitoring in state space", Journal of Process Control, vol. 13, no. 8, (2003), pp. 739-757.

[18] T. J. Harris, F. Boudreau and J. F. Macgregor, "Performance assessment of multivariable feedback controllers", Automatica, vol. 32, no. 11, (1996), pp. 1505-1518.

[19]B. Ko and T. F. Edgar, "Performance assessment of multivariable feedback control systems", Automatica, vol. 37, (2001), pp. 899-905.

[20] C. A. McNabb and S. J. Qin, "Projection based MIMO control performance monitoring: II -measured disturbances and setpoint changes", Journal of Process Control, vol. 15, no. 1, (2005), pp. 89-192.

[21] B. Huang, S. L. Shah and H. Fuji, "The unitary interactor matrix and its estimation using closed-loop data", Journal of Process Control, vol. 7, no. 3, (1997), pp. 195-207.

[22] Y. Peng and M. Kinnaert, "Explicit solution to the singular LQ regulation problem", IEEE Dransactions on Automatic Control, vol. 37, no. 5, (1992), pp. 633-636.

[23] S. Lu, "Multivariate process and control monitoring - Practical approaches and algorithnis", Master's thesis, University of Alberta, Edmonton, AB, Canada, (2005).

[24] B. Huang and S. L. Shah, "Practical issues in multivariable feedback control performance assessment", Journal of Process Control, vol. 8, no. 5-6, (1998), pp. 421 430.

[25] J. Yu and S. J. Qin, "MIMO control performance monit/ring using left/rightdiagonal interactors", Journal of Process Control, vol. 19, no. 8, (2009), pp. 1267-1276.
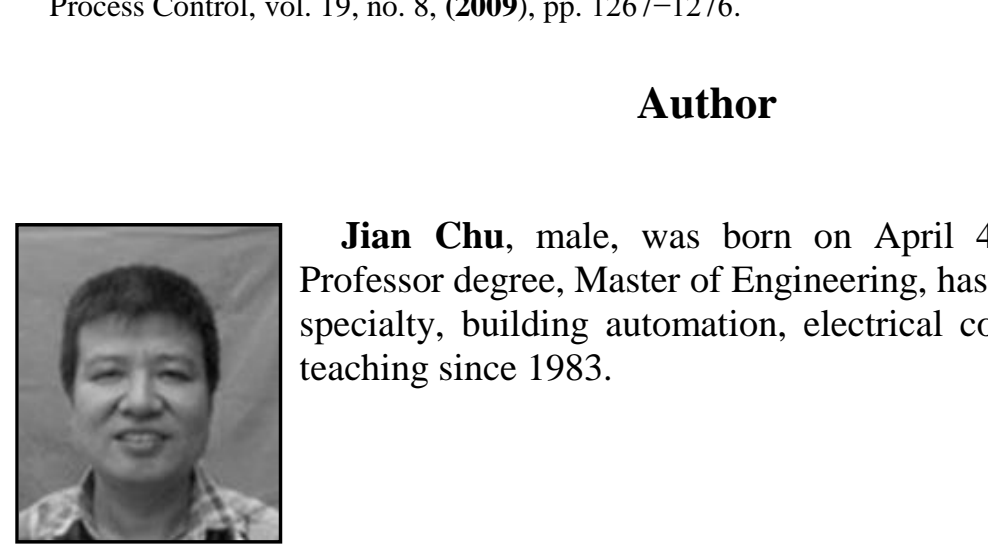

Jian Chu male, wâs born on April 4, 1961, in Tianjin, China. Professor degree, Master of Engineering, has been engaged in automation specialty, building automation, electrical controls and sensors work of teaching since 1983.

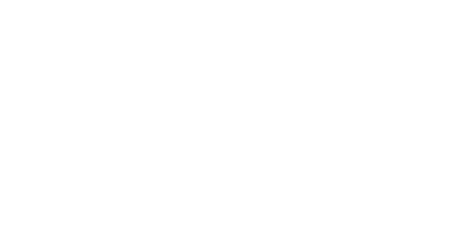

